\title{
RELATIVISTIC MANY-ELECTRON SCF CORRELATION DIAGRAM FOR SUPERHEAVY QUASIMOLECULES: $\mathrm{Pb}-\mathrm{Pb}$ 负
}

\author{
W.-D. SEPP, B. FRICKE and T. MOROVIĆ \\ Physics Department, University of Kassel, D-3500 Kassel, Germany
}

Received 14 May 1980

Revised manuscript received 4 November 1980

\begin{abstract}
We present the first relativistic many-electron SCF correlation diagram for a superheavy quasimolecule: $\mathrm{Pb}-\mathrm{Pb}$. The discussion shows a large number of quantitative as well as qualitative differences as compared with the known one-electron correlation diagram.
\end{abstract}

Realistic relativistic many-electron correlation diagrams are very important for the physical understanding and quantitative interpretation of the electronic processes occurring in adiabatic heavy ion collisions, because extended coupled channel calculations within a complete set of (in principle arbitrary) basis functions are not (yet) possible. The energy eigenvalues (and eigenfunctions) given as a function of the internuclear distance $R$ in the correlation diagram make it possible to discuss approximate physically adapted solutions of the time-dependent Schrödinger equation with these molecular basis functions which are expected to be relatively near to physical reality for medium and large internuclear distances $R$. Even in the region $R \leqq 100$ $\mathrm{fm}$, where in a high energetic collision most of the nonadiabatic effects occur, these basis functions are expected to be somewhat better (but also more "expensive") than those from the one-electron correlation diagrams.

Up to now for very heavy systems with a united charge $Z_{1}+Z_{2}>120$ there exist only one-electron correlation diagrams (except for the very asymmetrical system I + Au) [1]. As we will see below, screening effects are very important even for the innnermost levels of very heavy quasimolecules. Therefore a full relativistic self-consistent correlation diagram taking into account a realistic large number of electrons leads to a number of important qualitative and quantitative

\footnotetext{
Supported by the Gesellschaft fur Schwerionenforschung, Darmstadt, Germany.
}

changes as compared with the old one-electron correlation diagram [2], which are discussed below.

In fig. 1 we present our SCF correlation diagram for $\mathrm{Pb}-\mathrm{Pb}$ with 96 electrons on a double-logarithmic scale. It is calculated within a Dirac-Fock-Slater procedure [3] using a very large number of numerical DFS atomic basis functions of lead and additionally those of the united atom. The important improvements which were absolutely necessary in these calculations to avoid spurious states was the preorthogonalization of the basis so that linear dependencies could be omitted.

A careful comparison with the one-electron correlation diagram of Kirsch et al. [2] shows a number of significant differences and physically important changes:

(a) Even the strongest bound electronic levels are considerably screened. At $R=50 \mathrm{fm}$, the binding is lower by $8 \%$ for the $1(1 / 2)_{\mathrm{g}}, 25 \%$ for the $1(1 / 2)_{\mathrm{u}}$ and $40 \%$ for the $3(1 / 2)_{\mathrm{g}}$ level. At $R=500 \mathrm{fm}$, these changes are already $13 \%, 34 \%$, and $50 \%$, respectively. These numbers are a comparison between the exact one-electron and our many-electron calculations. Both calculations can be approximated with relatively good accuracy for very small internuclear distances by the so-called monopole approximation introduced by Soff et al. [4]. Of course, in a calculation such as a coupled channels calculation with the inclusion of outer crossings, binding energies and matrix elements have to be evaluated with a molecular many-electron calculation.

(b) Due to the change in screening at small internuclear distances the lowest levels which correlate to 


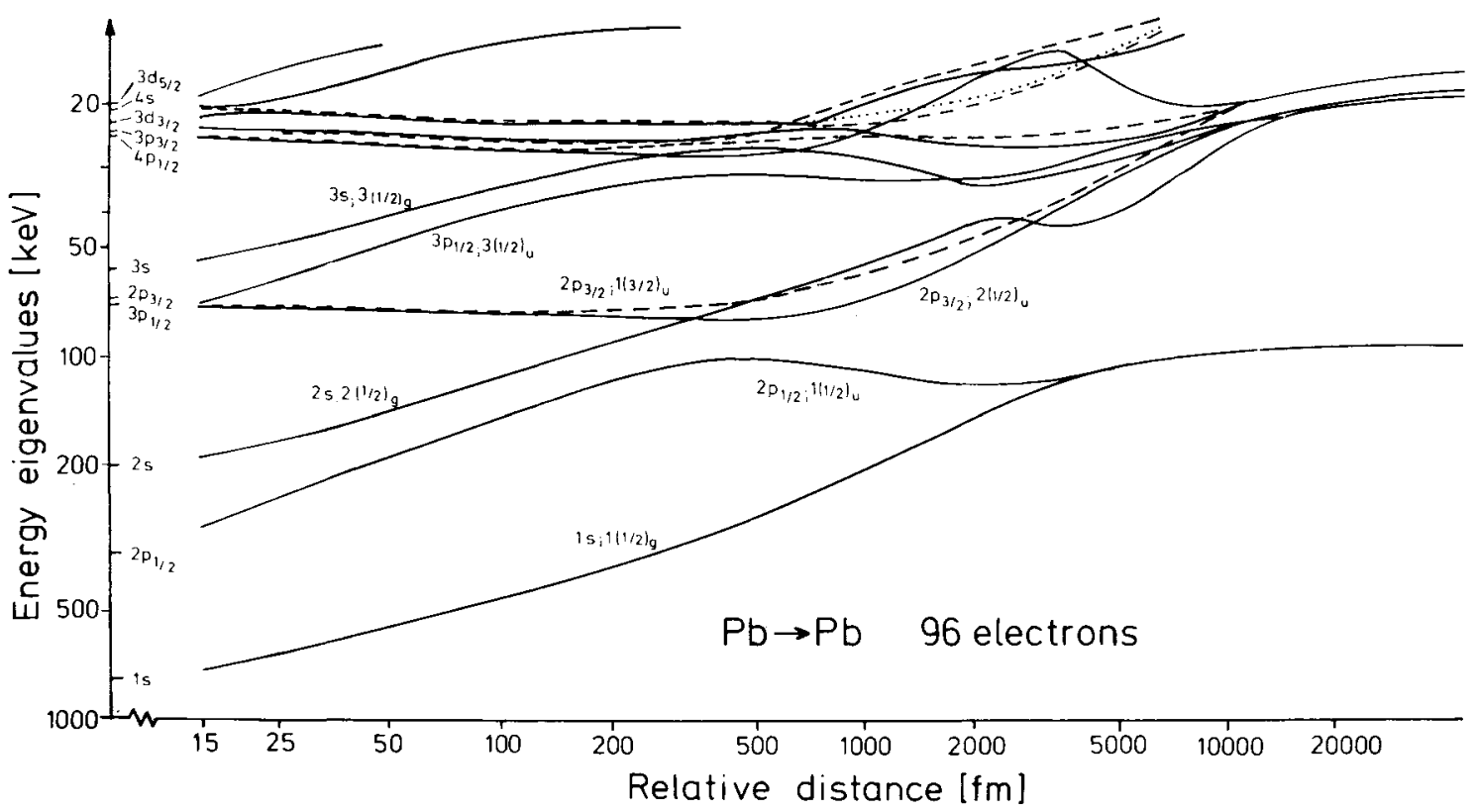

Fig. 1. Adiabatic correlation diagram for $\mathrm{Pb}-\mathrm{Pb}$ with 96 electrons calculated with a self-consistent-field relativistic Dirac-FockSlater method. The labels of the lowest levels show on the left the united atomic state and on the right the molecular label in the relativistic nomenclature.

$j=1 / 2$ atomic levels decrease even steeper at $R \rightarrow 0$ as compared with the one-electron correlation diagram.

(c) The $1(1 / 2)_{\mathrm{u}}$ level (which non-relativistically is called $2 \mathrm{p}_{\sigma}$ ) shows a very pronounced minimum at $R \approx$ $2000 \mathrm{fm}$, the energy eigenvalue being about $120 \mathrm{keV}$. This minimum does not exist in the one-electron correlation diagram of Kirsch et al. [2]. It only shows a region where the $2 \mathrm{p}_{\sigma}$ level is relatively constant in energy. This clear minimum in the many-electron correlation diagram is very important because Stoller et al. [5] observe an anisotropy maximum near $130 \mathrm{keV}$ in $\mathrm{Pb}-\mathrm{Pb}$ collisions. According to the interpretation of Gippner et al. [6] or Stoller et al. [7] such anisotropy maxima are strongly correlated with the minima in the correlation diagram. Proposals for a theoretical understanding of this effect have been given by Briggs et al. [8], Anholt [9] and Hartung et a1. [10]. The existence of the $2 \mathrm{p}_{\sigma}$ minimum is therefore a strong support for this interpretation which also seems to be valid even in the region of such heavy systems. In addition we find two further low lying isolated minima, one for the $2(1 / 2)_{\mathrm{g}}$ level at $R \approx 3800 \mathrm{fm}$ and another for the $3(1 / 2)_{\mathrm{u}}$ level at $R \approx 1000 \mathrm{fm}$. According to the interpretation stated above those minima may be correlated with the observed [11] anisotropy maxima at $40 \mathrm{keV}$ and 25 $\mathrm{keV}$, respectively.

(d) At small internuclear distances of about $500 \mathrm{fm}$ the many-electron correlation diagram shows pronounced minima for many levels correlated with atomic levels with $j>1 / 2$. We suppose that these minima are responsible for the additional anisotropy maxima, according to the interpretation stated in (c).

(e) The avoided crossing between the $1(1 / 2)_{\mathrm{u}}$ and $2(1 / 2)_{\mathrm{u}}$ levels is located at $R \approx 500 \mathrm{fm}$ with an energy difference $\Delta E \approx 20 \mathrm{keV}$. The corresponding values from the one-electron correlation diagrams are $R \approx$ $700 \mathrm{fm}$ and $\Delta E \approx 35 \mathrm{keV}$. A coupled channels calculation shows an increase of up to nearly an order of magnitude of the hole transfer when going from the oneelectron levels to the level structure in fig. 1. The actual increase of course is strongly dependent on the impact parameter and energy. The consequences are discussed in the next point.

(f) Due to the strong increase of the binding energy for all levels correlated with atomic levels with $j=1 / 2$ at very small internuclear distances, a non-negligible part of the holes could be transferred from the $M$ shell via the $\mathrm{L}$ shell into the $\mathrm{K}$ shell of $\mathrm{Pb}$ in a single colli- 
sion process. This is possible due to radial coupling between the $3(1 / 2)_{\mathrm{u}}$ level and the levels correlated with the $M$ shell. At very small distances the holes can be transferred from the $3(1 / 2)_{\mathrm{u}}$ level to the $2(1 / 2)_{\mathrm{u}}$ and $1(3 / 2)_{u}$ levels by radial and rotational coupling, respectively. Finally the crossing between the $2(1 / 2)_{u}$ and $1(1 / 2)_{\mathrm{u}}$ levels as discussed in (e) will allow a large hole transfer into the $\mathrm{K}$ shell of $\mathrm{Pb}$. This process is implicitly included in the paper of Soff et al. [12], but has not been discussed explicitly.

As a result one may say that such a realistic manyelectron correlation diagram which is presented here for the first time for a superheavy quasi-atomic system leads to a number of qualitative and quantitative changes in our understanding of the physics of inner shell phenomena in heavy ion scattering. Of course, a large number of additional detailed calculations have to be performed in the future.

\section{References}

[1] B. Fricke, T. Morovic, W.-D. Sepp, A. Rosén and D.E. Ellis, Phys. Lett. 59A (1976) 375.

[2] J. Kirsch et al., Phys. Lett. 72B (1978) 298.

[3] The general method is described in: A. Rosén and D.E. Ellis, J. Chem. Phys. 62 (1975) 3039.

[14] For an extended discussion see: G. Soff, J. Reinhardt, W. Betz and J. Rafelski, Phys. Scr. 17 (1978) 417.

[5] Ch. Stoller et al., Z. Phys., to be published.

[6] P. Gippner et al., Phys. Lett. 60B (1976) 249.

[7] Ch. Stoller, W. Wölfli, G. Bonani, M. Stöckli and M. Suter, J. Phys. B10 (1977) L347.

[8] J.S. Briggs and K. Dettmann, J. Phys. B10 (1977) 1113.

[9] R. Anholt, Z. Phys. A228 (1978) 257.

[10] H. Hartung and B. Fricke, Z. Phys. A228 (1978) 345.

[11] W. Wölfli, private communication.

[12] G. Soff, W. Greiner, W. Bets and B. Müller, Phys. Rev. A20 (1979) 169. 